\title{
Chronic Headache Education and Self- management Study (CHESS) - a mixed method feasibility study to inform the design of a randomised controlled trial
}

Kimberley White', Rachel Potter ${ }^{1 *}$, Shilpa Patel ${ }^{1}$, Vivien P. Nichols ${ }^{1}$, Kirstie L. Haywood ${ }^{2}$, Siew Wan Hee², Dipesh Mistry ${ }^{1}$, Dawn Carnes ${ }^{3}$, Stephanie J. C. Taylor ${ }^{4}$, Martin Underwood ${ }^{1}$, Manjit S. Matharu ${ }^{5}$ and on behalf of the CHESS team

\begin{abstract}
Background: Self-management support programmes are effective in a range of chronic conditions however there is limited evidence for their use in the treatment of chronic headaches. The aim of this study was to test the feasibility of four key aspects of a planned, future evaluative trial of a new education and self-management intervention for people with chronic headache: 1) recruiting people with chronic headache from primary care; 2) a telephone interview for the classification of chronic headaches; 3) the education and self-management intervention itself; and 4) the most appropriate patient reported outcomes (PROMS).

Methods: Participants were identified and recruited from general practices in the West Midlands of the UK. We developed a nurse-led chronic headache classification interview and assessed agreement with an interview with headache specialists. We developed and tested a group based education and self-management intervention to assess training and delivery receipt using observation, facilitator, and participant feedback. We explored the acceptability and relevance of PROMs using postal questionnaires, interviews and a smartphone app.

Results: Fourteen practices took part in the study and participant recruitment equated to 1.0/1000 registered patients. Challenges to recruitment were identified. We did 107 paired headache classification interviews. The level of agreement between nurse and doctor interviews was very good. We piloted the intervention in four groups with 18 participants. Qualitative feedback from participants and facilitators helped refine the intervention including shortening the overall intervention and increasing the facilitator training time. Participants completed 131 baseline questionnaires, measurement data quality, reliability and validity for headache-specific and generic measures was acceptable.
\end{abstract}

Conclusion: This study indicated that recruiting people with chronic headache from primary care is feasible but challenging, our headache classification interview is fit for purpose, our study intervention is viable, and that our choice of outcome measures is acceptable to participants in a future randomised controlled trial (RCT).

Trial registration: ISRCTN, ISRCTN79708100. Registered 16th December 2015, http://www.isrctn.com/ ISRCTN79708100

Keywords: Chronic headache, Feasibility study, Self-management, Recruitment, Outcome measures, Primary care

\footnotetext{
* Correspondence: r.potter@warwick.ac.uk

${ }^{1}$ Warwick Clinical Trials Unit, Warwick Medical School, University of Warwick,

Coventry CV4 7AL, UK

Full list of author information is available at the end of the article
}

(c) The Author(s). 2019 Open Access This article is distributed under the terms of the Creative Commons Attribution 4.0 International License (http://creativecommons.org/licenses/by/4.0/), which permits unrestricted use, distribution, and reproduction in any medium, provided you give appropriate credit to the original author(s) and the source, provide a link to the Creative Commons license, and indicate if changes were made. The Creative Commons Public Domain Dedication waiver (http://creativecommons.org/publicdomain/zero/1.0/) applies to the data made available in this article, unless otherwise stated. 


\section{Background}

Self-management support programmes have an established place in the management of a range of chronic diseases [1], however evidence for self-management programmes for use in chronic headaches disorders is currently limited [2]. The National Institute for Health Research (NIHR) funded a programme of work (RP-PG1212-20,018) to develop, and test, a non-pharmacological approach for chronic headache using education and self-management. NIHR programme grants fund research for conditions that cause substantial disease burden and usually consist of 'an interrelated group of high quality projects focused on a coherent theme, requiring multidisciplinary approaches, including clinical, health economics, statistics, qualitative and behavioural sciences, to ensure that research objectives can be met'. [3] Here we report the findings from a feasibility study we completed as part of our programme of work in preparation for a randomised controlled trial (RCT) evaluating the effectiveness of the intervention.

We wanted to test the feasibility of four key aspects prior the planned trial. Firstly, we wanted to test the feasibility of recruiting people with chronic headache from primary care and estimate the population base needed to recruit enough participants for the trial. Nearly a fifth of trials in 2011 were terminated for not meeting sufficient recruitment targets, and therefore unable to answer their research questions meaningfully [4].

Secondly, we needed to be able to classify common chronic headaches in participants identified from primary care. Specifically we wanted to test the feasibility of using a telephone classification interview that can be used by a non-headache specialist to classify the common chronic headache disorders: chronic migraine, chronic tension type headache (TTH) and medication overuse headache $(\mathrm{MOH})$. Many people with chronic headache disorders do not have an accurate diagnosis and receive inappropriate treatment of their headaches [5]. We wanted the classification interview to allow classification of headache type for both reporting and analysis purposes and to be used as part of the study intervention to allow targeted, individualised, treatment and advice. A systematic review failed to identify a simple classification tool fit for our purpose, we therefore needed to develop and validate a tool which can be used by a non-headache specialist to classify common chronic headache disorders [6].

Thirdly, we wanted to test the feasibility of developing and delivering the education and self-management support intervention for the management of common chronic headache disorders and examine the acceptability of the intervention to participants. Evaluations of complex interventions can be undermined by problems of acceptability, compliance and delivery of interventions [7].
Finally, we wanted to test the quality, acceptability and appropriateness of patient reported outcome measures (PROMs) for the trial. The selection of appropriate outcomes is crucial to the design of a trial and outcomes need to be relevant to people with chronic headaches [8]. A systematic review of the quality and acceptability of patientreported outcome measure highlighted the paucity of good quality PROM evaluations in this population and the limited focus on measurement relevance and acceptability to end-users, that is, people with headache [9]. We therefore wanted to understand which outcomes are important and relevant to people with chronic headache, a population who are often young adults with work and family commitments. Additionally, electronic diaries have shown to be acceptable to participants and may have the advantage of reducing recall effects $[10,11]$; we wanted to test the feasibility of using a smartphone app to collect weekly data on headache frequency, duration and severity.

\section{Methods}

This feasibility study was designed to determine what can be done, what should be done and how it can be done well for a future RCT [12]. It was a mixed method study to test and evaluate the feasibility of a newly developed education and self-management intervention for chronic headaches, future trial recruitment methods and the most appropriate outcome measures. It included, in addition, an embedded reliability study for the classification of headaches disorders, reported in more detail elsewhere [13]. The components of the feasibility study are shown in Fig. 1. We did not conduct a full pilot trial but chose to test the feasibility of four crucial components of the main randomised controlled trial due to the complexity and importance of each of these components. The study ran from January 2016 to April 2017.

\section{Patient and public involvement}

Patient and public involvement (PPI) was built into the key stages of the feasibility study to ensure that the research focused on issues that were important and relevant to patients and the public [14]. At the start of the study we established a lay advisory group of people with chronic headache to work with collaboratively. We identified members of the group from Universities/User Teaching and Research Action Partnership (UNTRAP) at the University of Warwick and sent out an advert to our three partner headache groups: Migraine Trust, Migraine Action and National Migraine Centre (In 2018 Migraine Action merged with Migraine Trust). The CHESS Lay Advisory Group specifically supported our application for ethical approval for the study, development of the headache classification interview, development of the study intervention and the choice of patient reported outcome measures. 


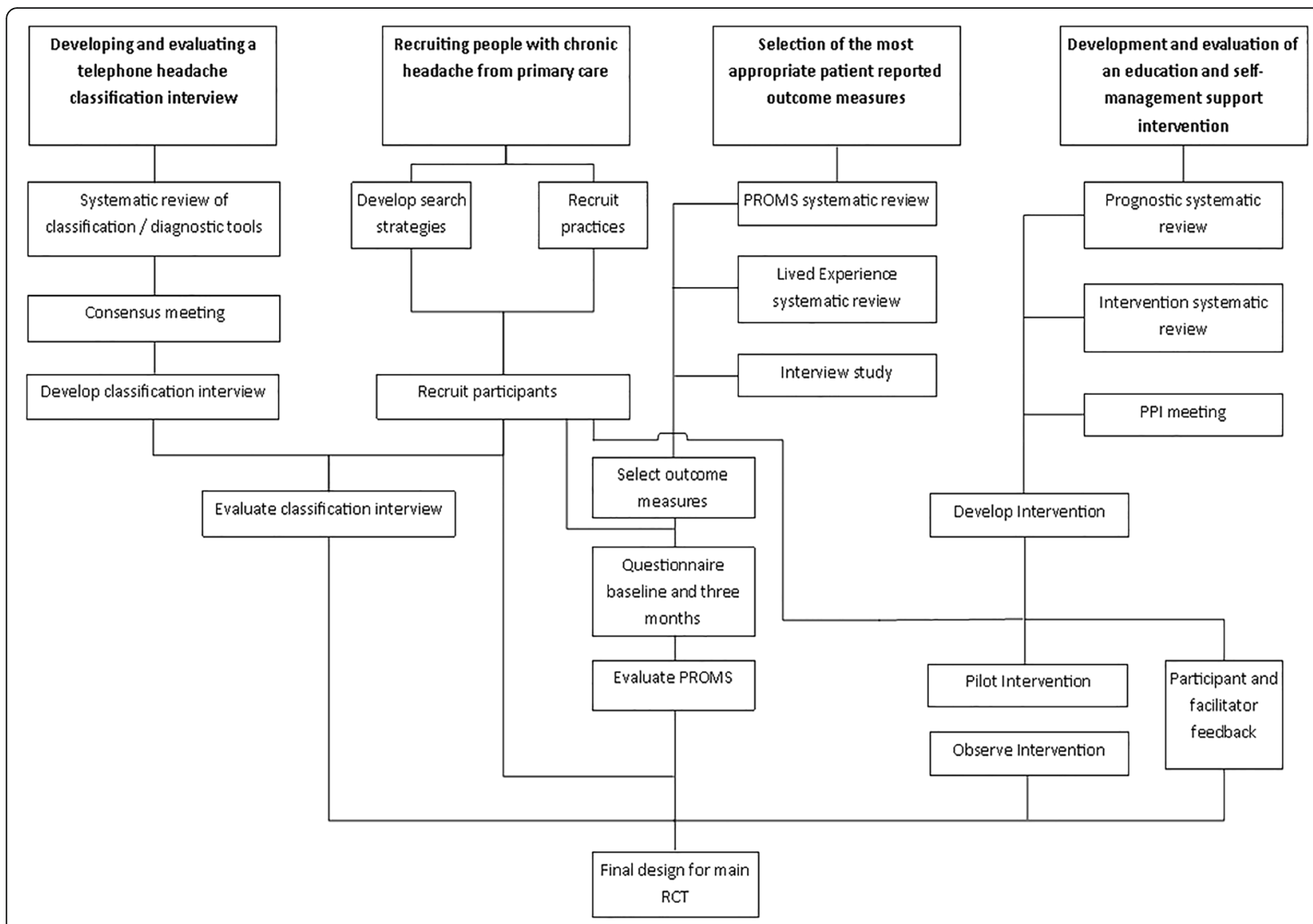

Fig. 1 Components of the Feasibility Study

\section{Feasibility of recruiting people with chronic headache from primary care}

The aim of this part of the study was to test the feasibility of our recruitment procedures and recruit a sample of participants to test the telephone headache classification interview, to pilot the education and self-management intervention, and to test the feasibility and the outcomes measures.

\section{Setting}

We aimed to recruit patients with chronic headache registered with general (family) practices in the West Midlands region of the UK. We ran the study in three clinical commissioning groups (CCGs) in the West Midlands which cover urban, small town and semirural areas with varying levels of deprivation and ethnic diversity. We initially ran the study in five Clinical Research Network (CRN) West Midlands South 'host practices' with extensive research experience. Subsequently we purposively selected additional practices to maximise diversity and to fill groups for the pilot intervention. We sought feedback via email using a short structured questionnaire from a small sample of General Practitioners (GPs) from the participating practices to explore their experience of taking part in the study.

\section{Participants}

The eligibility criteria for the feasibility study were:

\section{Inclusion Criteria:}

1. Aged $\geq 18$ years with chronic headache; defined as headache for 15 or more days per month for at least 3 months.

2. Able and willing to comply with the study procedures and provide written consent.

3. Fluent in written and spoken English.

\section{Exclusion Criteria:}

1) Has an underlying serious psychiatric or psychological disorder that precludes participation in the group intervention.

2) Known secondary cause of headache other than medication overuse headache; e.g.: primary or secondary brain tumour.

3) No access to a telephone. 
4) Currently participating in another clinical trial (with an unregistered medicinal product), or less than 90 days have passed since completing participation in such a trial.

The recruitment process to identify people for the study involved a standardised electronic search for general practice databases using Read-codes [15]. Initial scoping work indicated this standard clinical terminology system for coding chronic headache was rarely used, we therefore devised a search strategy to identify patients aged $\geq 18$ years who had consulted with headache (migraine, TTH and medication overuse headache) or had been prescribed migraine specific drugs (i.e. triptans, pizotifen) in the preceding 12 months. GPs then screened the list for patients it would be inappropriate to approach e.g. poorly controlled serious mental illness, terminal illness, or known secondary causes of headache other than medication overuse headache.

Potentially eligible patients were invited to participate in the study by a letter from their GP which also included a patient information leaflet informing them about the study. We also designed a study poster for display in patient waiting areas. People interested in the study were invited to contact the study team and asked the following questions to confirm eligibility:

1. On average how many days in the month do you get headaches?

2. How long have you been having your headaches this frequently for?

3. Has this been for at least the last 3 months?

4. Are you currently taking part in a drug trial?

Patients who met the eligibility criteria were informed that they would be asked to complete two telephone headache classification calls (one by a nurse the second by a headache specialist doctor) and that they may be invited to attend the education and self-management programme and/or take part in the interview study. Potential participants also had the opportunity to have any questions answered regarding the study.

Baseline packs were sent to people who were eligible and interested in the study, they included a consent form, a baseline questionnaire and a freepost return envelope. If necessary, a reminder pack was sent after 2 weeks. All participants were asked to provide written consent to complete postal questionnaires, a Smartphone App and the two telephone classification interviews. Study entry was marked by receipt of the signed consent form.

\section{Sample size}

For the suite of work in the feasibility study we initially sought to recruit 170 people with chronic headaches from primary care. The driver for this sample size was to have sufficient data to allow us to assess the inter-rater reliability of the telephone classification interview when done by two raters; namely a nurse, and a doctor experienced in headache management. We assumed level of agreement to be 0.8 , a substantial agreement [16]. The initial sample size was based on measuring the level of agreement for the classification of migraine (yes/no), TTH (yes/no) and $\mathrm{MOH}$ (yes/no). Following our systematic review of diagnostic tools and our classification consensus meeting, the outcomes from the classification changed to measuring the level of agreement in the classification of definite chronic migraine, probable chronic migraine and chronic TTH as well as presence or absence of $\mathrm{MOH}$ as a nominal scale. As the analyses changed from three pairwise comparisons to two pairwise comparisons, the multiplicity adjustment also changed hence giving a revised sample size target of 153 paired interviews which was approved by the programme steering committee and the funder [17].

An initial pilot search suggested that around 30/1000 people registered with a GP consult for headaches (acute, episodic or chronic) annually. Assuming that a third of these consulters had chronic headaches and a quarter of these joined the feasibility study recruitment rate would be $2.5 / 1000$ or $8.3 \%$ of those identified as consulting with headaches. Based on an average practice population of 7000 we estimated we needed 6-10 practices with a combined list size of 64,000 people to recruit our sample.

\section{Feasibility of a telephone classification interview to classify common headache disorders}

We developed a telephone headache classification interview for use by a non-headache specialist to classify chronic headache types for reporting and analysis purposes and that could also be used as part of the study intervention to allow targeted treatment and advice. In brief, we did a systematic literature review to identify any existing tools used to classify or diagnose different headache types which was presented to delegates at a headache classification consensus conference attended by headache specialists and people with chronic headache [6]. At the consensus conference delegates agreed what were the important questions to include in the classification interview. The classification interview was not intended to have a rigid interview structure or set questions, instead the person conducting the interview was encouraged to use a logic model to inform their clinical reasoning and decision-making.

We aimed to test the feasibility of training nurses to use the classification interview to classify chronic headache 
disorders and test the reliability of the tool. To validate the classification interview we trained six nurses, all non-headache experts, to conduct the interviews. The training included a one-day workshop delivered by a neurologist specialised in headache plus time with a member of the study team to practice classification interviews using mock scenarios and a training manual.

Participants from the feasibility study were interviewed first by the nurse and later by a doctor from the National Migraine Centre. The doctor classification was the assumed 'gold standard'. Participants were classified into: definite chronic migraine, probable chronic migraine or chronic TTH (with or without medication overuse) or 'other' headache type (other chronic primary headache or suspected secondary headache). We measured level of agreement between the classifications by nurses and doctors by using simple kappa statistics and prevalenceadjusted bias-adjusted kappa (PABAK).

The development and evaluation of the telephone headache classification interview is described in detail elsewhere [13].

Feasibility and acceptability of the education and selfmanagement support intervention for chronic headache

We developed the education and self-management intervention using the Medical Research Council (MRC) framework for complex interventions [7]. Development was informed by three systematic reviews 1.prognostic factors in chronic headache [18], 2. education and self-management interventions for chronic headache [19] and 3. the lived experiences of chronic headache. We drew from the experience of a previously tested self-management intervention for chronic pain [20] and we did qualitative interviews with people with chronic headache to inform the intervention design. The qualitative interviews were with members of the charity Migraine Action to gain their views on what was important to include in the education and self-management intervention. We held a collaborative intervention design meeting, attended by headache specialist clinicians, headache charity representatives, lay people with chronic headache, psychologists, and researchers.

The education and self-management intervention was intended to be delivered in a group format (8-10 per group) facilitated by a nurse and a lay person (with chronic headache). Topics included in the intervention were: understanding headache mechanisms, medication management, mood and headache, recognising unhelpful thought patterns and behaviours, stress management, sleep management, communication and mindfulness. The two and a half day programme used a range of methods including: group discussions, sharing narratives and experiences, problem solving, watching an educational DVD, role play and taster sessions. This was followed by a one to one consultation with a nurse to classify their headache type and discuss medication, lifestyle factors and goal setting, and up to 8 weeks of telephone support.

The development of the education and selfmanagement interventions is described in detail elsewhere [21].

We aimed to test the feasibility of the new intervention by running four groups each with up to 10 participants in community settings. We approached people who lived within easy travelling distance of proposed groups; participants provided written consent to attend the group intervention. We wanted to recruit and train two lay people and three nurses to deliver the intervention. The acceptability of the intervention was explored by conducting qualitative interviews with the participants who attended the groups and the facilitators that delivered the groups. Thematic analysis was used to identify common themes across the different components of the intervention.

\section{Feasibility of the patient reported outcome measures}

We proposed that our primary outcome measure for the RCT would be a headache-specific outcome measure collected by postal questionnaire. We initially did a systematic review of the quality and acceptability of patient reported outcome measures for episodic and chronic headache disorders [9], and a qualitative review of the lived experience of chronic headache [22] to understand what outcomes are important to people with chronic headache. This process supported the short-listing of both headache-specific (Migraine-Specific Questionnaire v2.1(MSQv2.1) [23] and the Headache Impact Test 6-item (HIT-6) [24] and generic measures (EuroQoL EQ-5D-5 L) [25] and Short-Form 12-item Health Status questionnaire (SF-12) [26] to include in the feasibility study. However, the migraine-specificity of the MSQv2.1 [23] made it unsuitable for use with our chronic headache population. Therefore, with permission from the developers, the target attribute of 'migraine' was changed to 'headache' and the questionnaire renamed as the 'Chronic Headache Quality of Life Questionnaire' (CHQLQv1.0). We evaluated both the acceptability and psychometric performance (data quality, reliability, validity) of the modified measure against the HIT-6, EQ-5D-5 L and SF-12 [24-26], providing the first evidence for the performance of the CHQLQ and HIT-6 [24] in a UK population and supporting selection for the RCT. Structured cognitive interviews were also conducted to explore the acceptability and relevance of the measures. Informed by good practice guidance, the interviews explored how responder's made judgements when completing the PROMs, including aspects such as question comprehension, recall and ease of completion $[27,28]$. The cognitive interviews and their analysis was 
carried out by an experienced qualitative team with expertise in this area.

\section{Data collection}

All participants were asked to complete postal questionnaires with the selected measures CHQLQ, HIT-6, SF-12 and EQ-5D-5 L at baseline (the point of consent) and at 2 weeks and 12 weeks after the baseline questionnaire was returned. The study team posted the questionnaire with a covering letter and a freepost return envelope. After 1 week if the questionnaire had not been received a reminder was sent and, 1 week following the reminder a telephone call would be made if the questionnaire was not received.

A smartphone application (app) compatible with IPhones, IPads and Android devices was designed by Clinvivo Ltd. for use in the study. The app asked participants to complete three simple questions regarding the frequency, severity and duration of the headaches they experienced. The questions were developed with the involvement of the CHESS Lay Advisory Group. The app requested the data to be completed weekly for up to 12 weeks and provided notification reminders for those who accepted this option. A small number of participants were approached to test the app; these were all participants who had recently agreed to take part in the study at the time the app was ready for testing.

\section{Results}

Feasibility of recruiting people with chronic headache from primary care

Practice recruitment

We recruited 14 general practices with a combined practice population of 128,634 (range 3300 to 16,886), see Fig. 2. Feedback from the short structured email questionnaire to GPs indicated that practices were mainly interested in the study because they felt a self-management programme

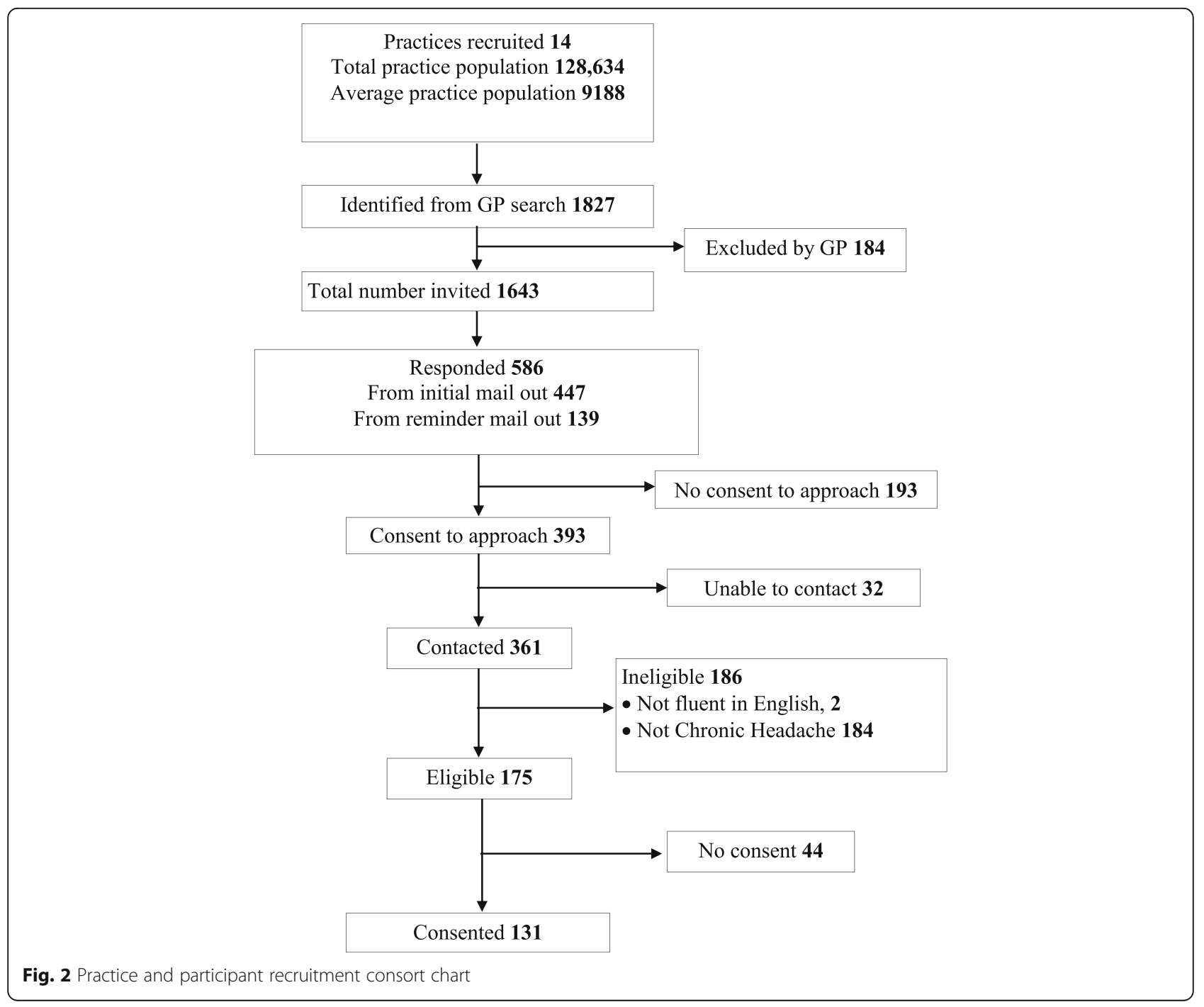


could potentially provide a useful alternative option for the management of patients with frequent headaches.

\section{Participant recruitment}

Searches of general practice data bases identified 1827 potential participants (14.2/1000 of registered patients). GPs excluded 184 (10\%) of these as inappropriate to approach. The remaining 1634 (1.3\% of total list size) were invited to take part in the study. We received 586 (36\%) responses, of these 393 (24\%) were interested in being contacted by the study team; 193 were not interested in the study. We succeeded in contacting 361/393 (92\%) often after numerous attempts to get hold of people; of these potential participants 175 (48\% of those contacted, $11 \%$ of those 1634 invited) were eligible. We received valid consent forms from $75 \%$ (131/175) of eligible participants (8\% of those 1634 invited). Forty people failed to respond and four formally withdrew at this stage. We recruited 1.0/1000 of practice list size.

Participants mean age was 49 years (range 21-77, standard deviation, SD, 13.3). There were 108 (82\%) female participants, 125 (95\%) of white ethnicity and 86 (66\%) in full or part-time employment. About one third ( $n=47,36 \%$ ) left full time education between age 17 and 19 , and another third $(n=44,34 \%)$ left full time education after 20 years old (Table 1 ).

\section{Feasibility of the headache classification interview}

We trained six research nurses to conduct the telephone classification interviews. Feedback from the training indicated that the nurses felt that the training workshop, opportunity to practice interviews and the training manual prepared them adequately to carry out the classification calls and that they gained confidence the more interviews they completed. Nurses and doctors from the NMC completed 111 and 108 headache classifications interviews respectively. There were 107 paired interviews. Median days between interviews was 32 (interquartile range, IQR, 21 to 48 days). Proportion of concordance of agreement between nurses' and doctors' interviews was 0.91 , with moderate or very good agreement on PABAK agreement in main and sensitivity analyses respectively. Full details of these analyses are reported elsewhere [13].

\section{Feasibility of the education and self-management support intervention}

We approached 85 participants to pilot the education and self-management programme; we were unable to contact 12 (14\%) participants and 46 (54\%) participants were unable to attend, reasons included work commitments, dates being unsuitable, home life (including childcare) and holidays (Fig. 3), 27 (32\%) expressed interest in attending the intervention and of these 18 (21\%) provided written consent to attend a group.

We piloted the CHESS intervention in four groups and with a total of 18 participants. The attendance at groups ranged from 3 to 6 participants and 17 participants attended the one-to-one consultation with the nurse. Qualitative interviews were completed with 12 participants using topic guides to explore participants' experience of taking part in the intervention. On the whole the groups were considered acceptable and participants found the educational and self-management components useful and interesting and found the opportunity to meet with other people with chronic headache particularly helpful. Based on participant feedback we removed the half day follow-up session because participants found the time commitment too great and we included the sessions on communication and managing setbacks at the end of day two of the programme.

Facilitators gave us feedback in a focus group or interviews with the use of topic guides, including their experiences of delivering the intervention and the training received. They reported that they did not find the twoday training adequate time to cover the delivery of the group intervention and the headache classification and medication information for the one-to-one consultations. It was also difficult for the lay facilitators to commit to delivering the intervention due to existing work and family commitments and unpredictability of their own headaches.

\section{Feasibility of the patient reported outcome measures}

Participants completed and returned 131 baseline questionnaires; 115 (88\%) and 103 (79\%) questionnaires were returned at two and 12-week follow up respectively. Measurement data quality, reliability and validity for the headache-specific and generic measures was reached at acceptable standards [29, 30], supporting application of the measures with groups of patients with chronic headache. Participants in the cognitive interviews $(n=14)$ indicated items included in the CHQLQ were comprehensive in scope and particularly welcomed those referring to the emotional impact of headache, and found the measure easy to complete. The lack of recall period for the first three items of the HIT- 6 was a concern. The generic measures were considered to be acceptable.

In total eight participants downloaded the Smartphone App, participants completed the app for a duration of up to 11 weeks. A telephone call was made to a selection of participants to check they were happy using the app and although participants didn't report difficulties downloading or using the app only one participant completed all 11 weeks of data collection and only four participants completed half or more of the weeks. 
Table 1 Participant demographics

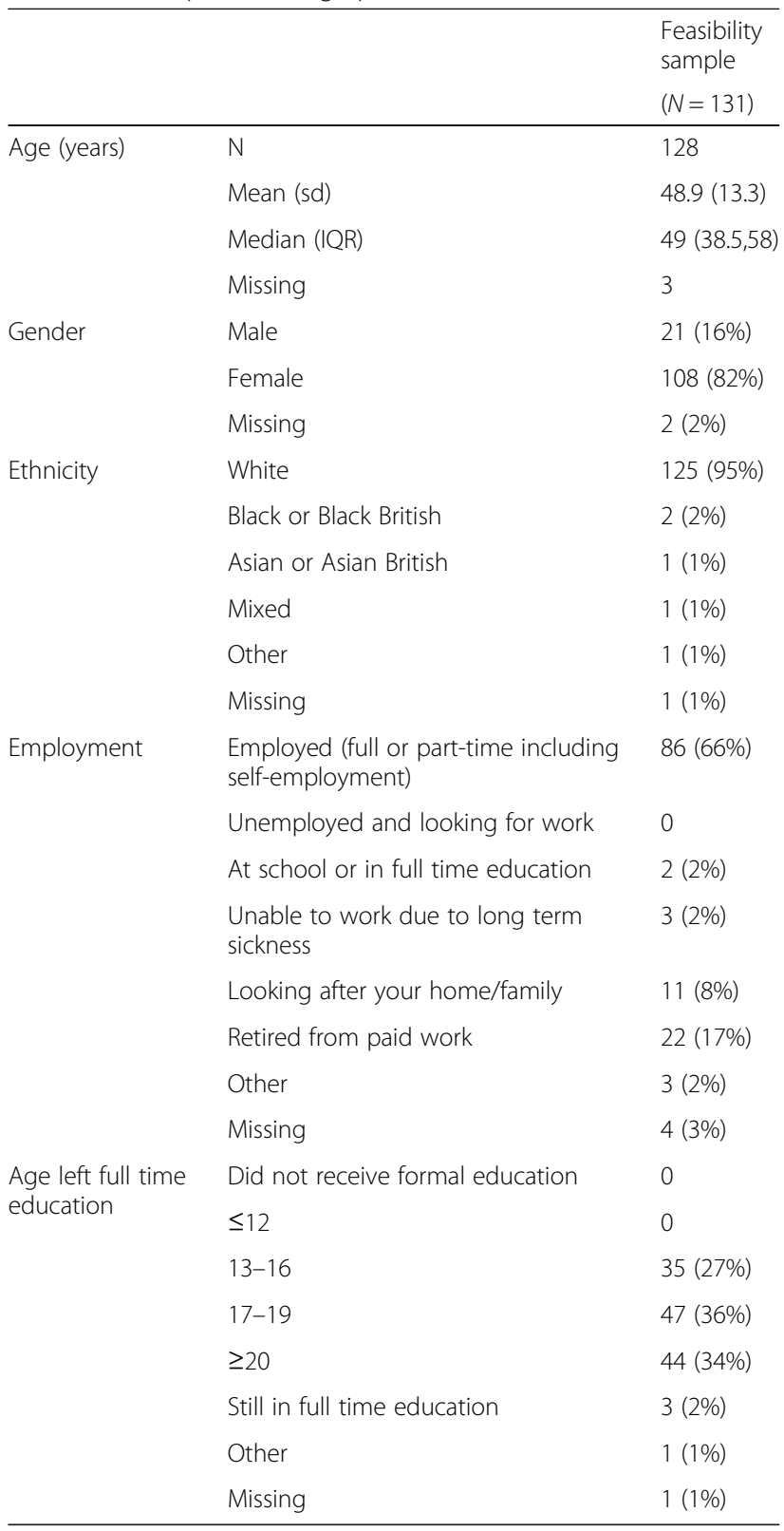

\section{Discussion}

One of the key objectives for the study was to test the feasibility of recruiting people with chronic headache from primary care and estimate the population base needed to recruit enough participants for the RCT. We successfully recruited 14 general practices to the study and feedback from GPs suggested that an invitation to participate in a randomised controlled trial is likely to be well received by general practices.

Recruitment to the study equated to around one per 1000 of the list size; this is comparable to recruitment rates from general practice for other studies of chronic pain $[20,31,32]$. It is, however, substantially less than our pre-study assumptions. The number of people with headaches across our pool of 14 practices was a little under half of that anticipated and the conversion rate of $7.1 \%$ from identification to consent was slightly less than pre-study assumptions. The highest identification rate was 18.3/1000 ranging down to 8.1/1000 (data not shown) suggesting that whilst there is great variability in coding of headache in practices our initial scoping searches were erroneous. Our conversion rate estimate was slightly optimistic and again there was a wide variability in conversion rate by practice (3.3 to $9.4 \%$, data not shown). Consequently a wide range in recruitment rate $(0.6 / 1000$ to $1.6 / 1000$, data not shown). This means we under-recruited against our original target and will need to recruit participants from over 100 practices for the RCT.

Overall we gained much useful information and experience from the recruitment processes for this feasibility study and we have made some important changes to our approach for the main study including allowing self-referral to the study from posters in pharmacies local to participating general practices and word of mouth media exposure. Contacting a largely young working population was challenging often requiring numerous attempts by telephone and email and a flexible approach to contacting people outside usual working hours. Only $75 \%$ of those eligible to take part returned signed consent forms despite chasing.

We had also not fully anticipated the challenges of making paired headache classification calls meaning we had data on fewer people than originally planned. Nevertheless we did obtain sufficient data to evaluate the agreement between nurse and doctor interviews. Nonheadache specialist nurses were able to use our logic model to classify chronic headaches types and identify medication overuse headache and the level of agreement with interviews by doctors specialised in headache was good, giving us confidence in the classification interview in the RCT.

We successfully piloted the intervention in four groups and gained valuable feedback from participants and facilitators. The length of the group intervention was reduced by half a day because participants, found it hard to commit more time due to work and family commitments. Nurse facilitators requested more training in order to feel confident in headache classification and medication advice, and an additional day training has been added in the RCT. The group intervention was originally designed to be facilitated by a health professional and lay person with chronic headache, a model which has previously been successful for the delivery of group interventions for chronic pain [20]. Because of the unpredictability of their own headaches it was not possible for the lay facilitators to commit to 


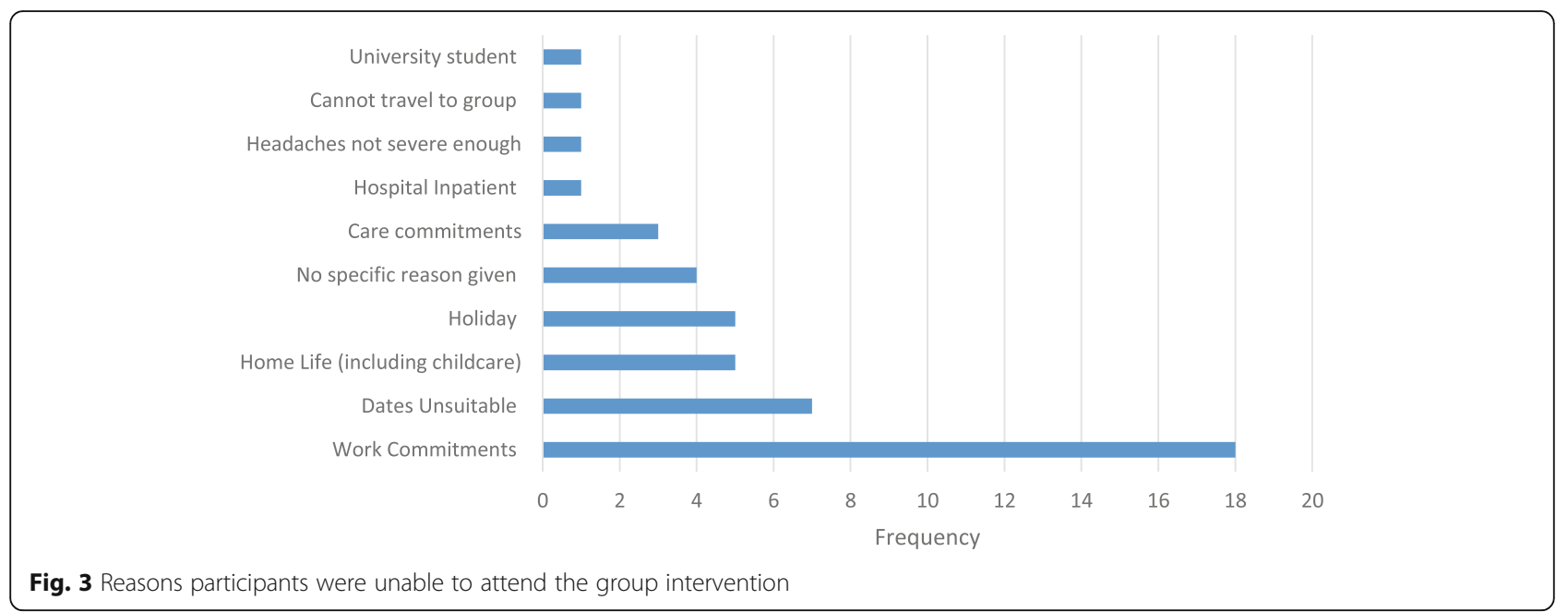

the role, and in the RCT the intervention will be facilitated by a nurse and an allied health professional. Alongside the RCT we will run a process evaluation to help understand how and if the intervention works. This will include collecting data on group attendance and interviews with a sample of participants and facilitators to explore the experience of delivering and receiving the intervention to inform any future roll out of the programme.

The completion and follow up of postal questionnaires was good, and all measures were well completed by responders at all time-points. Acceptable levels of data quality, reliability and validity were found for all measures, supporting their use with groups of people and justifying selection for the RCT. Participants indicated that the modified measure the Chronic Headache Quality of Life Questionnaire was both comprehensive and comprehensible. We were able to test our Smartphone App prior to the $\mathrm{RCT}$ in a small sample of participants, completion rates were poorer than anticipated and strategies to improve level of completion will be implemented in the main trial.

The advice and support of PPI was integral to the intervention development and other aspects of the feasibility study and the lay advisory group will continue their contribution into the main RCT.

The findings from the feasibility study have allowed us to be confident we are selecting the right participants and have a viable intervention, and allowed us to make an informed choice about outcome measures for the RCT. The feasibility study also identified challenges in recruitment of participants with chronic headache from primary care and collecting patient reported outcome measures that we have learnt from before starting the main trail.

The CHESS RCT (ISRCTN 79708100) which commenced January 2017 will test the effectiveness and cost effectiveness of the group education and self-management intervention compared with a best usual care and a relaxation $\mathrm{CD}$ for people living with chronic headaches (ISRCTN 79708100).

\section{Conclusions}

This study has demonstrated that recruiting people with chronic headache from primary care requires a large pool of patients which means recruiting many general practices and a flexible approach to contacting what is largely a young working population. We have developed and evaluated a telephone headache classification interview that can be used by a non-headache specialist to classify chronic headache disorders. We have provided essential evidence in support of a newly modified headache-specific measure, for application alongside established headache-specific and generic measures in this population. Despite our best efforts to involve lay people with chronic headache in the delivery of the intervention it was difficult due to their own person health; from a pragmatic stance the intervention was feasible when delivered by two health care practitioners.

\section{Abbreviations}

CHESS: Chronic Headache Education and Self-management Study;

CHQLQv1.0: Chronic headache quality of life questionnaire; GP: General Practitioner; HIT-6: Headache impact test 6-item; MOH: Medication overuse headache; MSQv2.1: Migraine-specific questionnaire v2.1; NIHR: National Institute for Health Research; PPI: Patient and public involvement; PROM: Patient reported outcome measure; RCT: Randomised controlled trial; SF-12: Short-form 12-item health status questionnaire; TTH: Tension type headache; UNTRAP: User teaching and research action partnership

\section{Acknowledgements}

We would like to acknowledge and thank all the participants that took part in the CHESS feasibility study. The manuscript was written on behalf of the CHESS co-applicants, study team and PPI representatives.

\section{Funding}

This research was funded by the NIHR Programme Grants for Applied Research programme (RP-PG-1212-20018). The views expressed in this publication are those of the author(s) and not necessarily those of the NHS, the NIHR or the Department of Health. 


\section{Availability of data and materials}

The datasets used and/or analysed during the current study are available from the corresponding author on reasonable request.

\section{Authors' contributions}

KW: acquisition and interpretation of data, study management and drafting of the manuscript. RP: study concept and design, acquisition and interpretation of data and drafting the manuscript. SP: study concept and design, acquisition and interpretation of data and critical revision of manuscript. VN: study design, acquisition and interpretation of data and critical revision of manuscript. KH: study concept and design, interpretation of data and critical revision of manuscript. SWH: study concept and design, analysis and interpretation of data and critical revision of manuscript. DM: study design, and analysis and interpretation of data and critical revision of manuscript. DC: study concept and design, interpretation of data and drafting of manuscript. SJT: study concept and design, interpretation of data and critical revision of manuscript. MU: project lead, study concept and design, interpretation of data and critical revision of manuscript. MM: study concept and design, interpretation of data and critical revision of manuscript. All authors read and approved the final manuscript.

\section{Ethics approval and consent to participate}

The study received Ethics approval from West Midlands - Black Country Research Ethics Committee (15/WM/0165). Participants provided written consent to take part in the study.

\section{Consent for publication}

N/A

\section{Competing interests}

MU was Chair of the NICE accreditation advisory committee until March 2017 for which he received a fee. He is chief investigator or co-investigator on multiple previous and current research grants from the UK National Institute for Health Research, Arthritis Research UK and is a co-investigator on grants funded by Arthritis Australia and Australian NHMRC. He has received travel expenses for speaking at conferences from the professional organisations hosting the conferences $\mathrm{He}$ is a director and shareholder of Clinvivo Ltd. that provides electronic data collection for health services research. Use of Clinvivo's App for this study was specified in original application for funding. MU has had no subsequent involvement in the tendering and contracting by University of Warwick and no involvement in the service provision by Clinvivo Ltd. related to this study. He is part of an academic partnership with Serco Ltd. related to return to work initiatives. He is an editor of the NIHR journal series for which he receives a fee. MM has served on the advisory board for Allergan, Medtronic, Novartis and Eli Lilly and has received payment for the development of educational presentations from Medtronic, Allergan and electroCore.

SP is a director of Health Psychology Services Ltd. which in part provides psychological treatments for those with chronic pain.

\section{Publisher's Note}

Springer Nature remains neutral with regard to jurisdictional claims in published maps and institutional affiliations.

\footnotetext{
Author details

'Warwick Clinical Trials Unit, Warwick Medical School, University of Warwick, Coventry CV4 7AL, UK. 'Division of Health Sciences, Warwick Medical School, University of Warwick, Coventry CV4 7A, UK. ${ }^{3}$ Faculty of Health, University of Applied Sciences, Fribourg, Western Switzerland, Switzerland. ${ }^{4}$ Centre for Primary Care and Public Health, Blizard Institute Barts and The London School of Medicine and Dentistry, Queen Mary University of London, London, UK. ${ }^{5}$ Headache Group, UCL Institute of Neurology and The National Hospital for Neurology and Neurosurgery, Queen Square, London WC1N 3BG, UK.
}

Received: 25 June 2018 Accepted: 31 January 2019

Published online: 11 February 2019

\section{References}

1. Taylor SJC, Pinnock H, Epiphaniou E, Pearce G, Parke HL, Schwappach A, et al. Health services and delivery research. A rapid synthesis of the evidence on interventions supporting self-management for people with long-term conditions: PRISMS - practical systematic review of selfmanagement support for long-term conditions. Southampton: NIHR Journals Library; 2014.

2. Carville S, Padhi S, Reason T, Underwood M. Diagnosis and management of headaches in young people and adults: summary of NICE guidance. BMJ. 2012;345:e5765.

3. National Institute for Health Research Programme Grants for Applied Research 2018 [Available from: https://www.nihr.ac.uk/funding-andsupport/funding-for-research-studies/funding-programmes/programmegrants-for-applied-research/.

4. Carlisle B, Kimmelman J, Ramsay T, Mackinnon N. Unsuccessful trial accrual and human subjects protections: an empirical analysis of recently closed trials. Clin Trials. 2015;12(1):77-83.

5. Kernick D, Stapley S, Hamilton W. GPs' classification of headache: is primary headache underdiagnosed? Br J Gen Pract. 2008;58(547):102-4

6. Potter R, Probyn K, Bernstein C, Pincus T, Underwood M, Matharu M. Diagnostic and classification tools for chronic headache disorders: a systematic review. Cephalalgia. 2018;333102418806864.

7. Craig P, Dieppe P, Macintyre S, Michie S, Nazareth I, Petticrew M. Developing and evaluating complex interventions: the new Medical Research Council guidance. BMJ (Clinical research ed). 2008;337:a1655.

8. Core Outcome Measures in Effectiveness Trials Initiative 2018 [Available from: http://www.comet-initiative.org/.

9. Haywood KL, Mars TS, Potter R, Patel S, Matharu M, Underwood M. Assessing the impact of headaches and the outcomes of treatment: a systematic review of patient-reported outcome measures (PROMs). Cephalalgia. 2017;333102417731348.

10. Burton C, Weller D, Sharpe M. Are electronic diaries useful for symptoms research? A systematic review. J Psychosom Res. 2007;62(5): 553-61.

11. Bolger N, Davis A, Rafaeli E. Diary methods: capturing life as it is lived. Annu Rev Psychol. 2003;54:579-616.

12. Eldridge $\mathrm{SM}$, Chan CL, Campbell MJ, Bond CM, Hopewell S, Thabane L, et al. CONSORT 2010 statement: extension to randomised pilot and feasibility trials. BMJ (Clinical research ed). 2016;355:15239.

13. Potter R, Hee SW, Griffiths F, Dodd K, Hoverd E, Underwood, et al. Development and validation of a telephone classification interview for common chronic headache disorders. J Headache Pain. 2019:20(2). https:// doi.org/10.1186/s10194-018-0954-z.

14. Staniszewska S, Brett J, Simera I, Seers K, Mockford C, Goodlad S, et al. GRIPP2 reporting checklists: tools to improve reporting of patient and public involvement in research. BMJ (Clinical research ed). 2017;358:j3453.

15. NHS Digital Read Codes [Available from: https:/digital.nhs.uk/data-andinformation/information-standards/terminology-and-classifications/read-codes.

16. Landis JR, Koch GG. The measurement of observer agreement for categorical data. Biometrics. 1977;33(1):159-74.

17. Donner A, Eliasziw M. A goodness-of-fit approach to inference procedures for the kappa statistic: confidence interval construction, significance-testing and sample size estimation. Stat Med. 1992;11(11):1511-9.

18. Probyn K, Bowers H, Caldwell F, Mistry D, Underwood M, Matharu M, et al Prognostic factors for chronic headache: a systematic review. Neurology. 2017:89(3):291-301.

19. Probyn K, Bowers H, Mistry D, Caldwell F, Underwood M, Patel S, et al. Nonpharmacological self-management for people living with migraine or tension-type headache: a systematic review including analysis of intervention components. BMJ Open. 2017;7(8):e016670.

20. Taylor SJC, Carnes D, Homer K, Kahan BC, Hounsome N, Eldridge S, et al. Novel three-day, community-based, nonpharmacological group intervention for chronic musculoskeletal pain (COPERS): a randomised clinical trial. PLoS Med. 2016;13(6):e1002040.

21. Patel S, Sandhu H, Pincus T, Taylor SJ, Underwood M, Matharu M. Development of an education and self-management intervention for chronic headache - CHESS trial (Chronic Headache Education and Selfmanagement Study). J Headache Pain. 2018; (submitted). 
22. Nichols VP, Ellard DR, Griffiths FE, Kamal A, Underwood M, Taylor SJC. The lived experience of chronic headache: a systematic review and synthesis of the qualitative literature. BMJ Open. 2017;7(12):e019929.

23. McKenna SP, Doward LC, Davey KM. The development and psychometric properties of the MSQOL: a migraine-specific quality-of-life instrument. Clin Drug Investig. 1998;15(5):413-23.

24. Kosinski M, Bayliss MS, Bjorner JB, Ware JE Jr, Garber WH, Batenhorst A, et al. A six-item short-form survey for measuring headache impact: the HIT-6. Qual Life Res. 2003;12(8):963-74.

25. Herdman M, Gudex C, Lloyd A, Janssen M, Kind P, Parkin D, et al. Development and preliminary testing of the new five-level version of EQ-5D (EQ-5D-5L). Qual Life Res. 2011;20(10):1727-36.

26. Jenkinson C, Layte R. Development and testing of the UK SF-12 (short form health survey). J Health Serv Res Policy. 1997;2(1):14-8.

27. Haywood KL, de Wit M, Staniszewska S, Morel T, Salek S. Developing patient-reported and relevant outcome measures. In: Facey KM, Ploug Hansen $\mathrm{H}$, Single ANV, editors. Patient involvement in health technology assessment. Singapore: Springer Singapore; 2017. p. 103-20

28. Hak T, Veer KVD, Jansen H. The Three-Step Test-Interview (TSTI): An observation-based method for pretesting self-completion questionnaires 2008. 2008;2(3):8\%J Survey Research Methods.

29. Terwee $\mathrm{CB}$, Bot SD, de Boer MR, van der Windt DA, Knol DL, Dekker J, et al. Quality criteria were proposed for measurement properties of health status questionnaires. J Clin Epidemiol. 2007;60(1):34-42.

30. Streiner D, Norman G, Cairney J. Health measurement scales: a practical guide to their development and use fifth ed. Oxford: Oxford University Press; 2014

31. United Kingdom back pain exercise and manipulation (UK BEAM) randomised trial. effectiveness of physical treatments for back pain in primary care. BMJ (Clinical research ed). 2004;329(7479):1377.

32. Lamb SE, Hansen Z, Lall R, Castelnuovo E, Withers EJ, Nichols V, et al. Group cognitive behavioural treatment for low-back pain in primary care: a randomised controlled trial and cost-effectiveness analysis. Lancet (London, England). 2010; 375(9718):916-23.

Ready to submit your research? Choose BMC and benefit from:

- fast, convenient online submission

- thorough peer review by experienced researchers in your field

- rapid publication on acceptance

- support for research data, including large and complex data types

- gold Open Access which fosters wider collaboration and increased citations

- maximum visibility for your research: over $100 \mathrm{M}$ website views per year

At $\mathrm{BMC}$, research is always in progress.

Learn more biomedcentral.com/submissions 